\title{
Predictive analysis of wettability of Al-Si based multiphase alloys and aluminum matrix composites by machine learning and physical modelling
}

\author{
Amir Kordijazi ${ }^{1,2}$, Swaroop Behera ${ }^{2}$, Dhrumil Patel ${ }^{2}$, Pradeep Rohatgi ${ }^{2}$, Michael Nosonovsky ${ }^{3}$ \\ Supporting Information
}

Tables and plots with data.



Figure S1. Variation of Contact Angle with wt\% Si. (All samples ground with 600 sand paper and 4ul drop size is used) Similar variation is seen with other sand paper grits and droplet sizes. 


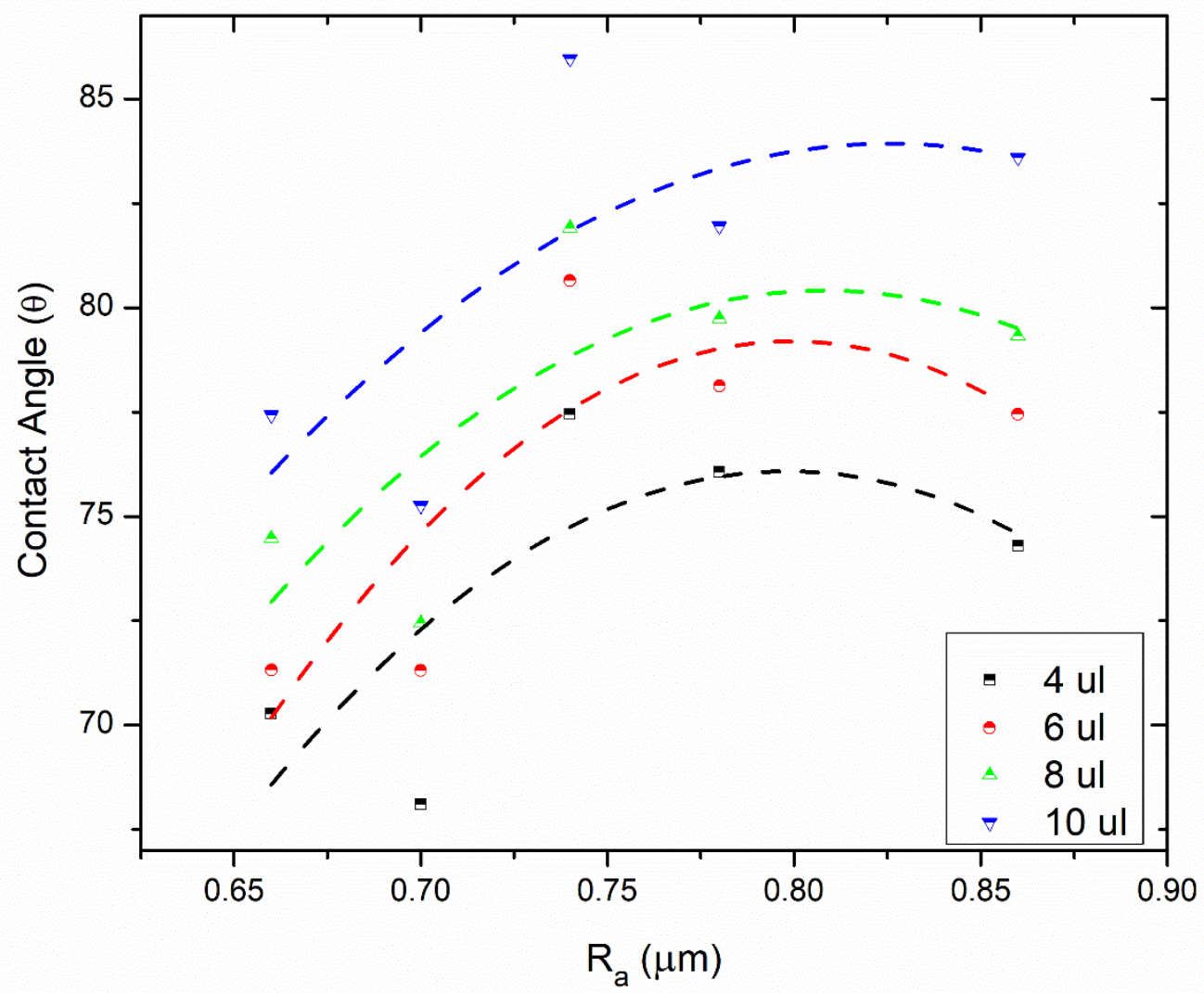

Figure S2. Variation of Contact Angle with roughness and droplet size (Sample - Al 7wt\% Si). 


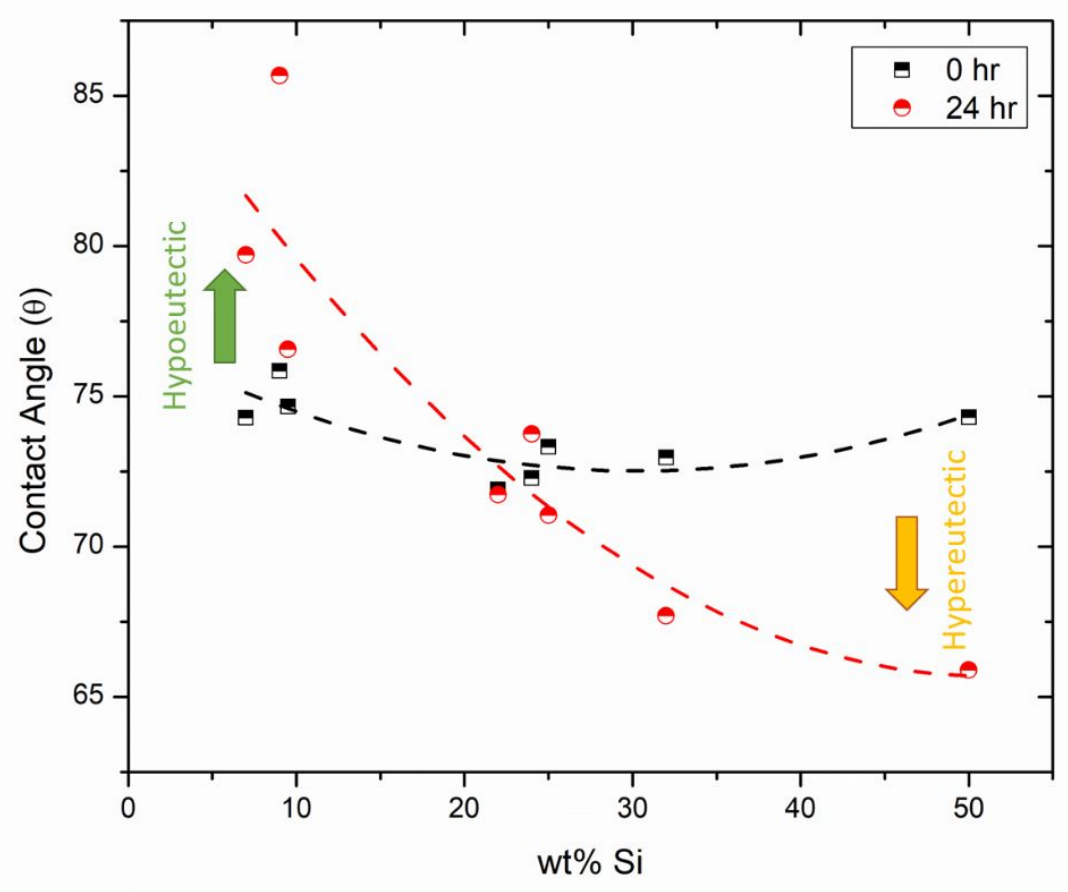

Figure S3. Variation of the CA with time.

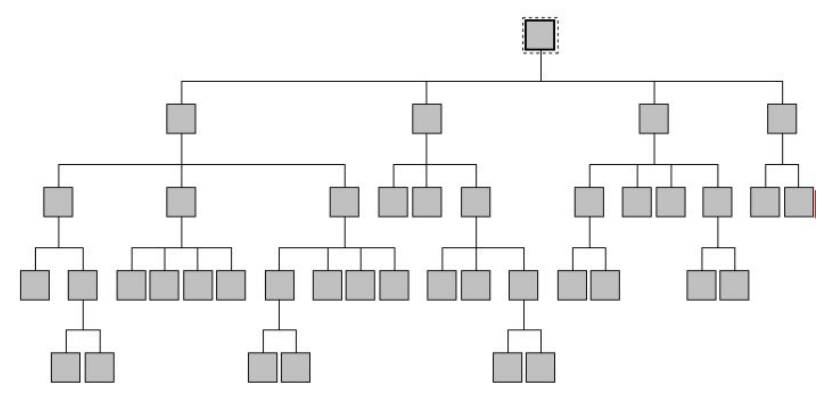

Figure S4. CHAID tree. 


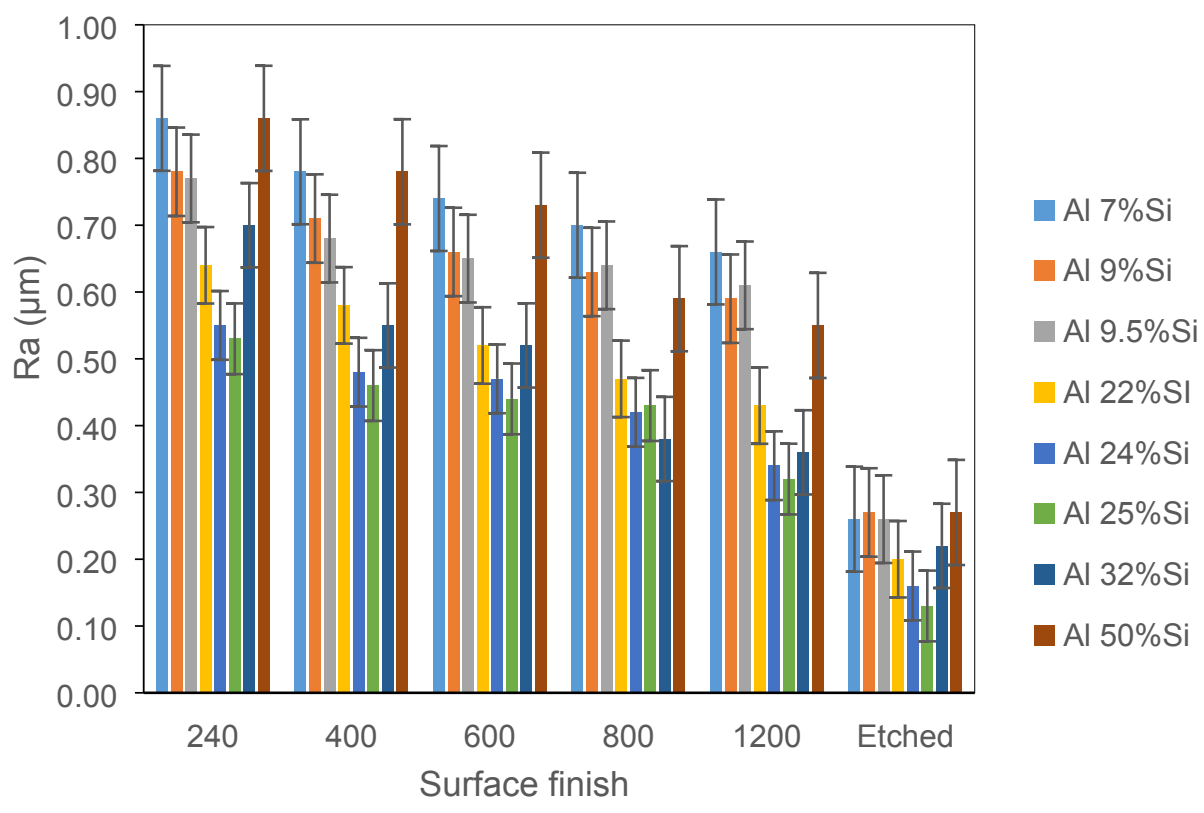

Figure S5. Surface roughness vs. surface finish. 

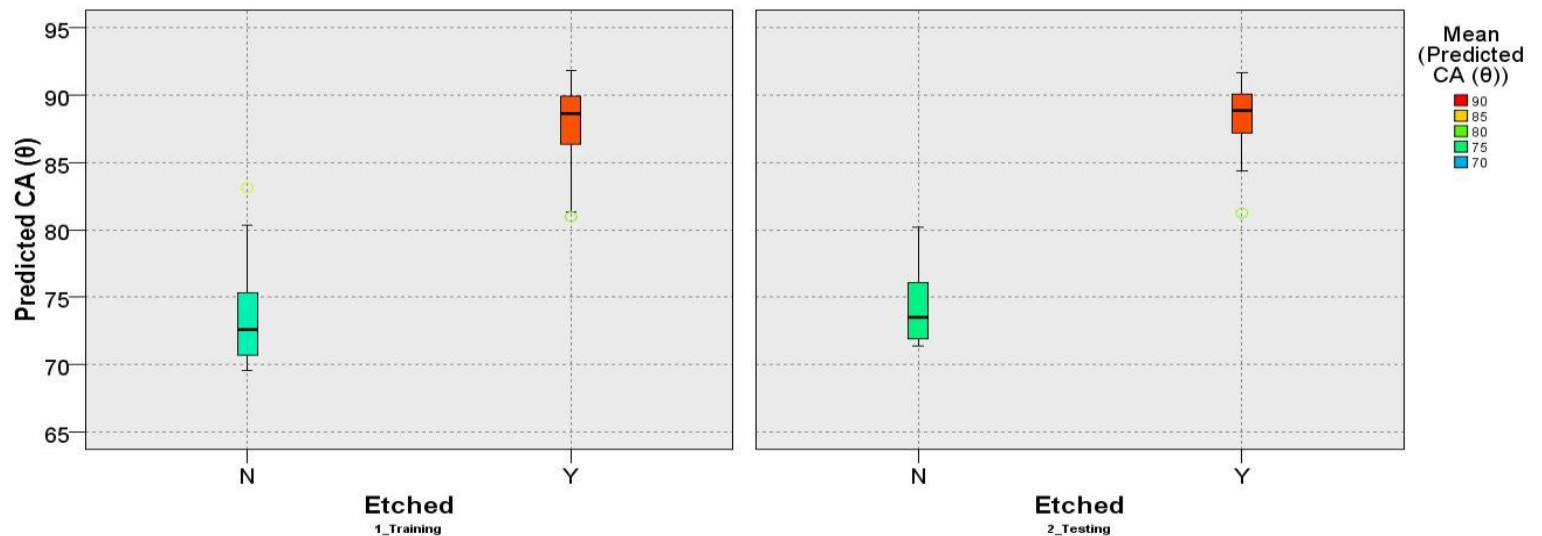

(a)

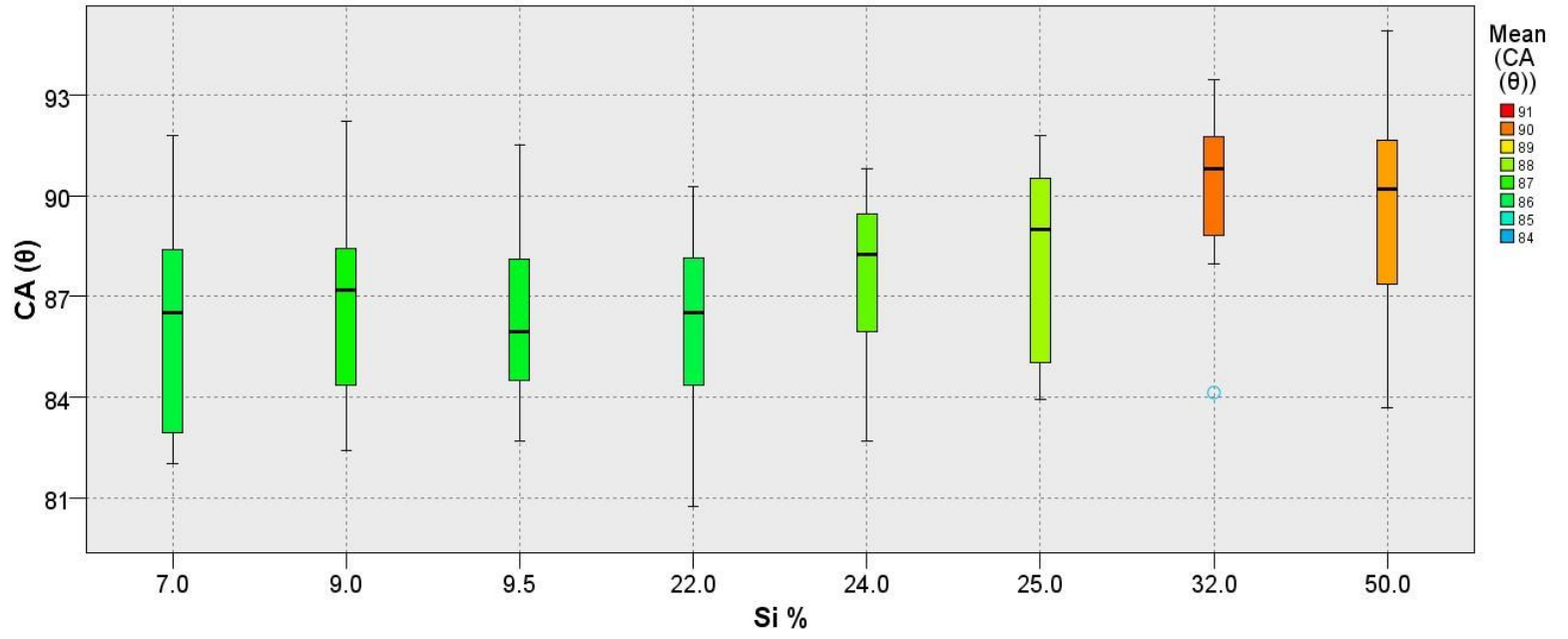

(b)

Figure S6. (a) Effect of etching on CA values for the training set and test set (b) Boxplots showing CA values as a function of $\mathrm{Si} \%$ for etched samples. 
(a)

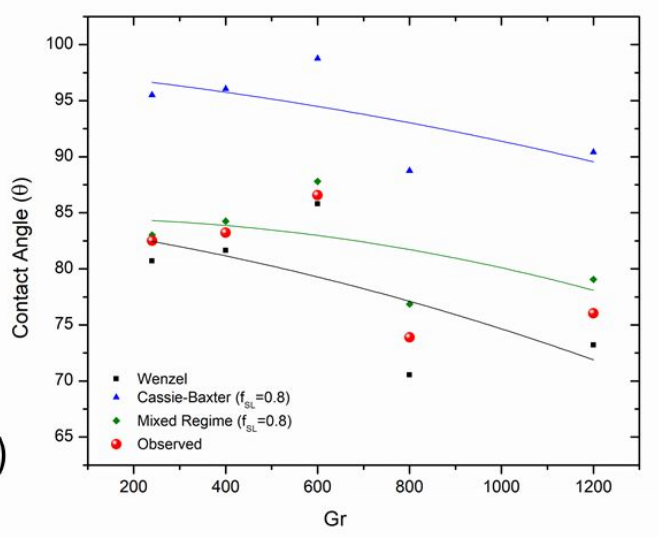

(b)

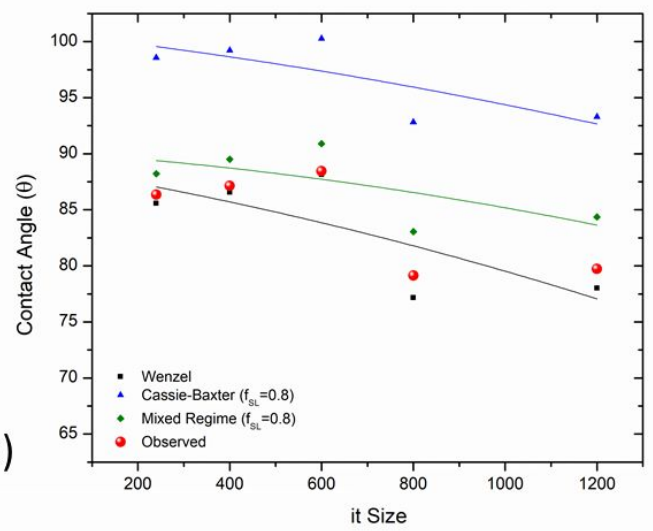

(c)

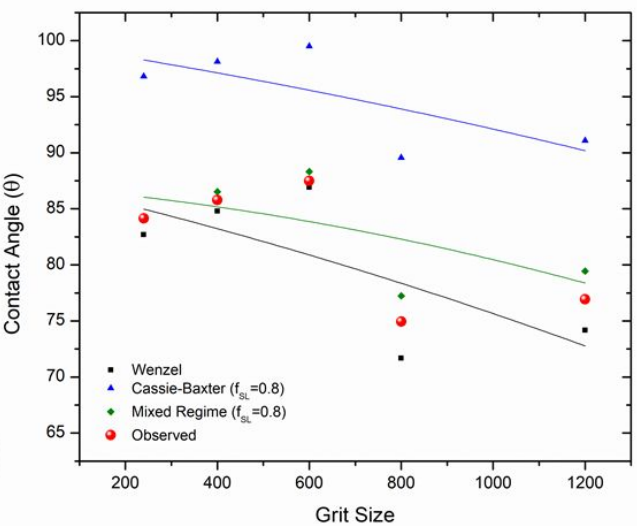

Figure S7. Plot of Wenzel CA, Cassie-Baxter CA and Mixed Regime CA and observed (measured) CA for composite samples (a) A356-10 SiC-5 Gr, (b) A356-5 NiAl $-5 \mathrm{Gr}$, (c) Al-16Si-5 NiAl $-5 \mathrm{Gr}$

Table S1. Composition of alloys and MMCs studied in this research

\begin{tabular}{c}
\hline Alloy \\
\hline$A l-7.0 \mathrm{Si}(\mathrm{Al356})$ \\
$\mathrm{Al}-9.0 \mathrm{Si}(\mathrm{Al360})$ \\
$\mathrm{Al}-9.5 \mathrm{Si}(\mathrm{Al368})$ \\
$\mathrm{Al}-22 \mathrm{Si}$ \\
$\mathrm{Al}-24 \mathrm{Si}$ \\
$\mathrm{Al}-25 \mathrm{Si}$ \\
$\mathrm{Al}-32 \mathrm{Si}$ \\
$\mathrm{Al}-50 \mathrm{Si}$ \\
$\mathrm{Al} 356-10 \mathrm{SiC}_{-}-5 \mathrm{Gr}$ \\
$\mathrm{Al} 356-5 \mathrm{NiAl}-5 \mathrm{Gr}$ \\
$\mathrm{Al}-16 \mathrm{Si}-5 \mathrm{NiAl}_{3}-5 \mathrm{Gr}$
\end{tabular}


Table S2. Experimental results

\begin{tabular}{|c|c|c|c|c|c|}
\hline $\mathrm{CA}(\boldsymbol{\theta})$ & $\begin{array}{l}\text { Grit } \\
\text { Size }\end{array}$ & $\begin{array}{l}\text { Droplet } \\
(\mu \mathrm{L})\end{array}$ & Size & Si \% & $\begin{array}{l}\text { Time } \\
\text { (hr) }\end{array}$ \\
\hline 74.28 & 240 & 4 & & 7 & 0 \\
\hline 77.45 & 240 & 6 & & 7 & 0 \\
\hline 79.32 & 240 & 8 & & 7 & 0 \\
\hline 83.60 & 240 & 10 & & 7 & 0 \\
\hline 76.05 & 400 & 4 & & 7 & 0 \\
\hline 78.13 & 400 & 6 & & 7 & 0 \\
\hline 79.73 & 400 & 8 & & 7 & 0 \\
\hline 81.96 & 400 & 10 & & 7 & 0 \\
\hline 77.45 & 600 & 4 & & 7 & 0 \\
\hline 80.65 & 600 & 6 & & 7 & 0 \\
\hline 81.91 & 600 & 8 & & 7 & 0 \\
\hline 85.97 & 600 & 10 & & 7 & 0 \\
\hline 68.10 & 800 & 4 & & 7 & 0 \\
\hline 71.31 & 800 & 6 & & 7 & 0 \\
\hline 72.44 & 800 & 8 & & 7 & 0 \\
\hline 75.26 & 800 & 10 & & 7 & 0 \\
\hline 70.26 & 1200 & 4 & & 7 & 0 \\
\hline 71.32 & 1200 & 6 & & 7 & 0 \\
\hline 74.47 & 1200 & 8 & & 7 & 0 \\
\hline 77.43 & 1200 & 10 & & 7 & 0 \\
\hline 75.85 & 240 & 4 & & 9 & 0 \\
\hline 78.74 & 240 & 6 & & 9 & 0 \\
\hline 81.16 & 240 & 8 & & 9 & 0 \\
\hline 81.96 & 240 & 10 & & 9 & 0 \\
\hline 76.31 & 400 & 4 & & 9 & 0 \\
\hline 77.65 & 400 & 6 & & 9 & 0 \\
\hline 80.81 & 400 & 8 & & 9 & 0 \\
\hline 81.29 & 400 & 10 & & 9 & 0 \\
\hline 78.13 & 600 & 4 & & 9 & 0 \\
\hline 81.05 & 600 & 6 & & 9 & 0 \\
\hline 83.11 & 600 & 8 & & 9 & 0 \\
\hline 86.29 & 600 & 10 & & 9 & 0 \\
\hline 68.31 & 800 & 4 & & 9 & 0 \\
\hline 69.19 & 800 & 6 & & 9 & 0 \\
\hline 72.99 & 800 & 8 & & 9 & 0 \\
\hline 76.36 & 800 & 10 & & 9 & 0 \\
\hline 71.94 & 1200 & 4 & & 9 & 0 \\
\hline 74.17 & 1200 & 6 & & 9 & 0 \\
\hline 75.61 & 1200 & 8 & & 9 & 0 \\
\hline
\end{tabular}




\begin{tabular}{|c|c|c|c|}
\hline 76.83 & 1200 & 10 & 9 \\
\hline 74.65 & 240 & 4 & 9.5 \\
\hline 77.66 & 240 & 6 & 9.5 \\
\hline 79.23 & 240 & 8 & 9.5 \\
\hline 81.99 & 240 & 10 & 9.5 \\
\hline 73.30 & 400 & 4 & 9.5 \\
\hline 76.94 & 400 & 6 & 9.5 \\
\hline 79.95 & 400 & 8 & 9.5 \\
\hline 82.46 & 400 & 10 & 9.5 \\
\hline 77.16 & 600 & 4 & 9.5 \\
\hline 81.25 & 600 & 6 & 9.5 \\
\hline 81.76 & 600 & 8 & 9.5 \\
\hline 83.62 & 600 & 10 & 9.5 \\
\hline 67.81 & 800 & 4 & 9.5 \\
\hline 69.55 & 800 & 6 & 9.5 \\
\hline 72.31 & 800 & 8 & 9.5 \\
\hline 74.71 & 800 & 10 & 9.5 \\
\hline 68.73 & 1200 & 4 & 9.5 \\
\hline 70.85 & 1200 & 6 & 9.5 \\
\hline 72.51 & 1200 & 8 & 9.5 \\
\hline 75.91 & 1200 & 10 & 9.5 \\
\hline 71.89 & 240 & 4 & 22 \\
\hline 73.77 & 240 & 6 & 22 \\
\hline 77.31 & 240 & 8 & 22 \\
\hline 76.08 & 240 & 10 & 22 \\
\hline 72.32 & 400 & 4 & 22 \\
\hline 72.92 & 400 & 6 & 22 \\
\hline 75.03 & 400 & 8 & 22 \\
\hline 76.12 & 400 & 10 & 22 \\
\hline 73.91 & 600 & 4 & 22 \\
\hline 76.11 & 600 & 6 & 22 \\
\hline 78.30 & 600 & 8 & 22 \\
\hline 79.80 & 600 & 10 & 22 \\
\hline 62.80 & 800 & 4 & 22 \\
\hline 65.13 & 800 & 6 & 22 \\
\hline 67.98 & 800 & 8 & 22 \\
\hline 70.88 & 800 & 10 & 22 \\
\hline 65.42 & 1200 & 4 & 22 \\
\hline 69.10 & 1200 & 6 & 22 \\
\hline 71.27 & 1200 & 8 & 22 \\
\hline 74.28 & 1200 & 10 & 22 \\
\hline 72.28 & 240 & 4 & 24 \\
\hline 74.89 & 240 & 6 & 24 \\
\hline
\end{tabular}




\begin{tabular}{|c|c|c|c|}
\hline 77.91 & 240 & 8 & 24 \\
\hline 79.99 & 240 & 10 & 24 \\
\hline 73.28 & 400 & 4 & 24 \\
\hline 76.64 & 400 & 6 & 24 \\
\hline 78.59 & 400 & 8 & 24 \\
\hline 78.80 & 400 & 10 & 24 \\
\hline 74.85 & 600 & 4 & 24 \\
\hline 79.32 & 600 & 6 & 24 \\
\hline 80.19 & 600 & 8 & 24 \\
\hline 81.33 & 600 & 10 & 24 \\
\hline 66.54 & 800 & 4 & 24 \\
\hline 65.86 & 800 & 6 & 24 \\
\hline 71.49 & 800 & 8 & 24 \\
\hline 70.77 & 800 & 10 & 24 \\
\hline 68.80 & 1200 & 4 & 24 \\
\hline 69.92 & 1200 & 6 & 24 \\
\hline 71.24 & 1200 & 8 & 24 \\
\hline 75.36 & 1200 & 10 & 24 \\
\hline 73.31 & 240 & 4 & 25 \\
\hline 77.08 & 240 & 6 & 25 \\
\hline 77.60 & 240 & 8 & 25 \\
\hline 80.06 & 240 & 10 & 25 \\
\hline 74.92 & 400 & 4 & 25 \\
\hline 75.79 & 400 & 6 & 25 \\
\hline 78.00 & 400 & 8 & 25 \\
\hline 80.72 & 400 & 10 & 25 \\
\hline 76.46 & 600 & 4 & 25 \\
\hline 79.87 & 600 & 6 & 25 \\
\hline 81.58 & 600 & 8 & 25 \\
\hline 83.28 & 600 & 10 & 25 \\
\hline 65.72 & 800 & 4 & 25 \\
\hline 69.25 & 800 & 6 & 25 \\
\hline 71.30 & 800 & 8 & 25 \\
\hline 72.96 & 800 & 10 & 25 \\
\hline 68.80 & 1200 & 4 & 25 \\
\hline 70.74 & 1200 & 6 & 25 \\
\hline 73.88 & 1200 & 8 & 25 \\
\hline 76.10 & 1200 & 10 & 25 \\
\hline 72.96 & 240 & 4 & 32 \\
\hline 79.39 & 240 & 6 & 32 \\
\hline 78.16 & 240 & 8 & 32 \\
\hline 81.17 & 240 & 10 & 32 \\
\hline 76.29 & 400 & 4 & 32 \\
\hline
\end{tabular}




\begin{tabular}{|c|c|c|c|c|}
\hline 77.12 & 400 & 6 & 32 & 0 \\
\hline 80.34 & 400 & 8 & 32 & 0 \\
\hline 80.48 & 400 & 10 & 32 & 0 \\
\hline 77.09 & 600 & 4 & 32 & 0 \\
\hline 78.84 & 600 & 6 & 32 & 0 \\
\hline 84.12 & 600 & 8 & 32 & 0 \\
\hline 85.67 & 600 & 10 & 32 & 0 \\
\hline 66.28 & 800 & 4 & 32 & 0 \\
\hline 70.81 & 800 & 6 & 32 & 0 \\
\hline 73.67 & 800 & 8 & 32 & 0 \\
\hline 75.57 & 800 & 10 & 32 & 0 \\
\hline 71.34 & 1200 & 4 & 32 & 0 \\
\hline 73.84 & 1200 & 6 & 32 & 0 \\
\hline 73.79 & 1200 & 8 & 32 & 0 \\
\hline 75.51 & 1200 & 10 & 32 & 0 \\
\hline 74.30 & 240 & 4 & 50 & 0 \\
\hline 75.79 & 240 & 6 & 50 & 0 \\
\hline 76.92 & 240 & 8 & 50 & 0 \\
\hline 78.75 & 240 & 10 & 50 & 0 \\
\hline 75.17 & 400 & 4 & 50 & 0 \\
\hline 76.89 & 400 & 6 & 50 & 0 \\
\hline 77.54 & 400 & 8 & 50 & 0 \\
\hline 80.87 & 400 & 10 & 50 & 0 \\
\hline 77.26 & 600 & 4 & 50 & 0 \\
\hline 77.85 & 600 & 6 & 50 & 0 \\
\hline 82.63 & 600 & 8 & 50 & 0 \\
\hline 83.21 & 600 & 10 & 50 & 0 \\
\hline 66.01 & 800 & 4 & 50 & 0 \\
\hline 69.89 & 800 & 6 & 50 & 0 \\
\hline 70.50 & 800 & 8 & 50 & 0 \\
\hline 71.33 & 800 & 10 & 50 & 0 \\
\hline 69.60 & 1200 & 4 & 50 & 0 \\
\hline 71.45 & 1200 & 6 & 50 & 0 \\
\hline 75.06 & 1200 & 8 & 50 & 0 \\
\hline 75.93 & 1200 & 10 & 50 & 0 \\
\hline 79.70 & 240 & 4 & 7 & 24 \\
\hline 80.12 & 240 & 6 & 7 & 24 \\
\hline 81.32 & 240 & 8 & 7 & 24 \\
\hline 82.02 & 240 & 10 & 7 & 24 \\
\hline 80.70 & 400 & 4 & 7 & 24 \\
\hline 81.10 & 400 & 6 & 7 & 24 \\
\hline 84.09 & 400 & 8 & 7 & 24 \\
\hline 84.63 & 400 & 10 & 7 & 24 \\
\hline
\end{tabular}




\begin{tabular}{|c|c|c|c|}
\hline 74.83 & 600 & 4 & 7 \\
\hline 81.34 & 600 & 6 & 7 \\
\hline 82.90 & 600 & 8 & 7 \\
\hline 83.56 & 600 & 10 & 7 \\
\hline 81.31 & 800 & 4 & 7 \\
\hline 84.09 & 800 & 6 & 7 \\
\hline 85.30 & 800 & 8 & 7 \\
\hline 81.50 & 800 & 10 & 7 \\
\hline 64.11 & 1200 & 4 & 7 \\
\hline 66.35 & 1200 & 6 & 7 \\
\hline 65.73 & 1200 & 8 & 7 \\
\hline 68.05 & 1200 & 10 & 7 \\
\hline 85.67 & 240 & 4 & 9 \\
\hline 82.05 & 240 & 6 & 9 \\
\hline 83.23 & 240 & 8 & 9 \\
\hline 79.89 & 240 & 10 & 9 \\
\hline 72.41 & 400 & 4 & 9 \\
\hline 77.87 & 400 & 6 & 9 \\
\hline 78.21 & 400 & 8 & 9 \\
\hline 79.50 & 400 & 10 & 9 \\
\hline 81.35 & 600 & 4 & 9 \\
\hline 85.15 & 600 & 6 & 9 \\
\hline 87.12 & 600 & 8 & 9 \\
\hline 90.42 & 600 & 10 & 9 \\
\hline 75.64 & 800 & 4 & 9 \\
\hline 77.27 & 800 & 6 & 9 \\
\hline 67.57 & 800 & 8 & 9 \\
\hline 67.81 & 800 & 10 & 9 \\
\hline 73.02 & 1200 & 4 & 9 \\
\hline 75.53 & 1200 & 6 & 9 \\
\hline 77.48 & 1200 & 8 & 9 \\
\hline 77.51 & 1200 & 10 & 9 \\
\hline 76.56 & 240 & 4 & 9.5 \\
\hline 77.07 & 240 & 6 & 9.5 \\
\hline 81.77 & 240 & 8 & 9.5 \\
\hline 86.94 & 240 & 10 & 9.5 \\
\hline 73.22 & 400 & 4 & 9.5 \\
\hline 79.08 & 400 & 6 & 9.5 \\
\hline 81.88 & 400 & 8 & 9.5 \\
\hline 84.26 & 400 & 10 & 9.5 \\
\hline 72.12 & 600 & 4 & 9.5 \\
\hline 76.38 & 600 & 6 & 9.5 \\
\hline 78.45 & 600 & 8 & 9.5 \\
\hline
\end{tabular}




\begin{tabular}{|c|c|c|c|}
\hline 82.45 & 600 & 10 & 9.5 \\
\hline 68.70 & 800 & 4 & 9.5 \\
\hline 74.28 & 800 & 6 & 9.5 \\
\hline 75.15 & 800 & 8 & 9.5 \\
\hline 78.78 & 800 & 10 & 9.5 \\
\hline 67.56 & 1200 & 4 & 9.5 \\
\hline 69.93 & 1200 & 6 & 9.5 \\
\hline 72.07 & 1200 & 8 & 9.5 \\
\hline 83.45 & 1200 & 10 & 9.5 \\
\hline 71.73 & 240 & 4 & 22 \\
\hline 74.66 & 240 & 6 & 22 \\
\hline 80.89 & 240 & 8 & 22 \\
\hline 77.34 & 240 & 10 & 22 \\
\hline 61.69 & 400 & 4 & 22 \\
\hline 69.15 & 400 & 6 & 22 \\
\hline 73.60 & 400 & 8 & 22 \\
\hline 76.29 & 400 & 10 & 22 \\
\hline 66.18 & 600 & 4 & 22 \\
\hline 74.85 & 600 & 6 & 22 \\
\hline 81.59 & 600 & 8 & 22 \\
\hline 84.34 & 600 & 10 & 22 \\
\hline 65.29 & 800 & 4 & 22 \\
\hline 73.27 & 800 & 6 & 22 \\
\hline 67.72 & 800 & 8 & 22 \\
\hline 69.99 & 800 & 10 & 22 \\
\hline 72.73 & 1200 & 4 & 22 \\
\hline 68.98 & 1200 & 6 & 22 \\
\hline 71.55 & 1200 & 8 & 22 \\
\hline 72.70 & 1200 & 10 & 22 \\
\hline 73.74 & 240 & 4 & 24 \\
\hline 77.13 & 240 & 6 & 24 \\
\hline 81.49 & 240 & 8 & 24 \\
\hline 82.91 & 240 & 10 & 24 \\
\hline 71.64 & 400 & 4 & 24 \\
\hline 74.33 & 400 & 6 & 24 \\
\hline 78.43 & 400 & 8 & 24 \\
\hline 76.05 & 400 & 10 & 24 \\
\hline 68.12 & 600 & 4 & 24 \\
\hline 73.28 & 600 & 6 & 24 \\
\hline 80.86 & 600 & 8 & 24 \\
\hline 80.72 & 600 & 10 & 24 \\
\hline 67.90 & 800 & 4 & 24 \\
\hline 67.40 & 800 & 6 & 24 \\
\hline
\end{tabular}




\begin{tabular}{|c|c|c|c|c|}
\hline 64.88 & 800 & 8 & 24 & 24 \\
\hline 64.90 & 800 & 10 & 24 & 24 \\
\hline 71.01 & 1200 & 4 & 24 & 24 \\
\hline 76.35 & 1200 & 6 & 24 & 24 \\
\hline 77.40 & 1200 & 8 & 24 & 24 \\
\hline 77.72 & 1200 & 10 & 24 & 24 \\
\hline 71.04 & 240 & 4 & 25 & 24 \\
\hline 79.60 & 240 & 6 & 25 & 24 \\
\hline 84.06 & 240 & 8 & 25 & 24 \\
\hline 80.77 & 240 & 10 & 25 & 24 \\
\hline 74.69 & 400 & 4 & 25 & 24 \\
\hline 81.26 & 400 & 6 & 25 & 24 \\
\hline 81.95 & 400 & 8 & 25 & 24 \\
\hline 83.15 & 400 & 10 & 25 & 24 \\
\hline 66.35 & 600 & 4 & 25 & 24 \\
\hline 71.06 & 600 & 6 & 25 & 24 \\
\hline 82.25 & 600 & 8 & 25 & 24 \\
\hline 87.07 & 600 & 10 & 25 & 24 \\
\hline 66.86 & 800 & 4 & 25 & 24 \\
\hline 64.56 & 800 & 6 & 25 & 24 \\
\hline 65.94 & 800 & 8 & 25 & 24 \\
\hline 61.30 & 800 & 10 & 25 & 24 \\
\hline 67.62 & 1200 & 4 & 25 & 24 \\
\hline 76.34 & 1200 & 6 & 25 & 24 \\
\hline 77.33 & 1200 & 8 & 25 & 24 \\
\hline 80.83 & 1200 & 10 & 25 & 24 \\
\hline 67.70 & 240 & 4 & 32 & 24 \\
\hline 73.90 & 240 & 6 & 32 & 24 \\
\hline 79.86 & 240 & 8 & 32 & 24 \\
\hline 84.23 & 240 & 10 & 32 & 24 \\
\hline 74.98 & 400 & 4 & 32 & 24 \\
\hline 76.28 & 400 & 6 & 32 & 24 \\
\hline 78.13 & 400 & 8 & 32 & 24 \\
\hline 79.91 & 400 & 10 & 32 & 24 \\
\hline 75.78 & 600 & 4 & 32 & 24 \\
\hline 85.37 & 600 & 6 & 32 & 24 \\
\hline 86.77 & 600 & 8 & 32 & 24 \\
\hline 86.98 & 600 & 10 & 32 & 24 \\
\hline 61.20 & 800 & 4 & 32 & 24 \\
\hline 63.66 & 800 & 6 & 32 & 24 \\
\hline 67.81 & 800 & 8 & 32 & 24 \\
\hline 64.69 & 800 & 10 & 32 & 24 \\
\hline 80.91 & 1200 & 4 & 32 & 24 \\
\hline
\end{tabular}




\begin{tabular}{lllll}
79.23 & 1200 & 6 & 32 & 24 \\
81.51 & 1200 & 8 & 32 & 24 \\
82.54 & 1200 & 10 & 32 & 24 \\
65.90 & 240 & 4 & 50 & 24 \\
66.55 & 240 & 6 & 50 & 24 \\
67.24 & 240 & 8 & 50 & 24 \\
68.06 & 240 & 10 & 50 & 24 \\
66.85 & 400 & 4 & 50 & 24 \\
67.29 & 400 & 6 & 50 & 24 \\
75.04 & 400 & 8 & 50 & 24 \\
78.16 & 400 & 10 & 50 & 24 \\
76.68 & 600 & 4 & 50 & 24 \\
83.56 & 600 & 6 & 50 & 24 \\
90.86 & 600 & 8 & 50 & 24 \\
91.91 & 600 & 10 & 50 & 24 \\
71.21 & 800 & 4 & 50 & 24 \\
72.30 & 800 & 6 & 50 & 24 \\
73.77 & 800 & 8 & 50 & 24 \\
76.02 & 800 & 10 & 50 & 24 \\
75.77 & 1200 & 4 & 50 & 24 \\
76.53 & 1200 & 6 & 50 & 24 \\
76.73 & 1200 & 8 & 50 & 24 \\
78.59 & 1200 & 10 & 50 & 24 \\
\hline
\end{tabular}

Table S3. Experimental results

\begin{tabular}{llllll}
\hline Grit & $\begin{array}{l}\text { Droplet } \\
\text { Size } \\
\text { Size }\end{array}$ & Si \% & $\begin{array}{l}\text { Time } \\
(\mathbf{h r})\end{array}$ & $\begin{array}{l}\text { Observed } \\
\text { CA }(\boldsymbol{\theta})\end{array}$ & $\begin{array}{l}\text { Predicted } \\
\text { CA }(\boldsymbol{\theta})\end{array}$ \\
\hline 240 & 4 & 25 & 0 & 73.313 & 72.674 \\
240 & 6 & 25 & 0 & 77.08 & 75.851 \\
240 & 8 & 25 & 0 & 77.6 & 78.384 \\
240 & 10 & 25 & 0 & 80.06 & 79.223 \\
400 & 4 & 25 & 0 & 74.917 & 73.083 \\
400 & 6 & 25 & 0 & 75.787 & 76.194 \\
400 & 8 & 25 & 0 & 78 & 78.757 \\
400 & 10 & 25 & 0 & 80.723 & 77.869 \\
600 & 4 & 25 & 0 & 76.46 & 75.077 \\
600 & 6 & 25 & 0 & 79.87 & 77.95 \\
600 & 8 & 25 & 0 & 81.58 & 79.708 \\
600 & 10 & 25 & 0 & 83.283 & 81.327
\end{tabular}




\begin{tabular}{|c|c|c|c|c|c|}
\hline 800 & 4 & 25 & 0 & 65.723 & 66.401 \\
\hline 800 & 6 & 25 & 0 & 69.247 & 66.005 \\
\hline 800 & 8 & 25 & 0 & 71.297 & 70.139 \\
\hline 800 & 10 & 25 & 0 & 72.963 & 70.778 \\
\hline 1200 & 4 & 25 & 0 & 68.803 & 69.562 \\
\hline 1200 & 6 & 25 & 0 & 70.743 & 70.462 \\
\hline 1200 & 8 & 25 & 0 & 73.88 & 71.751 \\
\hline 1200 & 10 & 25 & 0 & 76.097 & 75.018 \\
\hline 240 & 4 & 25 & 24 & 71.037 & 72.336 \\
\hline 240 & 6 & 25 & 24 & 79.6 & 77.076 \\
\hline 240 & 8 & 25 & 24 & 84.06 & 80.663 \\
\hline 240 & 10 & 25 & 24 & 80.77 & 80.747 \\
\hline 400 & 4 & 25 & 24 & 74.687 & 70.361 \\
\hline 400 & 6 & 25 & 24 & 81.257 & 75.17 \\
\hline 400 & 8 & 25 & 24 & 81.947 & 78.55 \\
\hline 400 & 10 & 25 & 24 & 83.153 & 76.988 \\
\hline 600 & 4 & 25 & 24 & 66.347 & 68.21 \\
\hline 600 & 6 & 25 & 24 & 71.057 & 75.712 \\
\hline 600 & 8 & 25 & 24 & 82.247 & 79.416 \\
\hline 600 & 10 & 25 & 24 & 87.07 & 82.084 \\
\hline 800 & 4 & 25 & 24 & 66.857 & 67.93 \\
\hline 800 & 6 & 25 & 24 & 64.56 & 67.99 \\
\hline 800 & 8 & 25 & 24 & 65.943 & 65.461 \\
\hline 800 & 10 & 25 & 24 & 61.297 & 66.424 \\
\hline 1200 & 4 & 25 & 24 & 67.617 & 71.61 \\
\hline 1200 & 6 & 25 & 24 & 76.34 & 73.681 \\
\hline 1200 & 8 & 25 & 24 & 77.33 & 76.263 \\
\hline 1200 & 10 & 25 & 24 & 80.833 & 77.969 \\
\hline
\end{tabular}

Table S4. Experimental results

\begin{tabular}{lllll}
\hline $\begin{array}{c}\text { Time } \\
(\mathbf{h r})\end{array}$ & Si \% & Etched & $\begin{array}{c}\text { Droplet size } \\
(\boldsymbol{\mu L})\end{array}$ & CA (⿻) \\
\hline 0 & 7 & $\mathrm{~N}$ & 4 & 70.26333 \\
0 & 7 & $\mathrm{~N}$ & 6 & 71.32 \\
0 & 7 & $\mathrm{~N}$ & 8 & 74.46667 \\
0 & 7 & $\mathrm{~N}$ & 10 & 77.42667 \\
0 & 7 & $\mathrm{Y}$ & 4 & 83.62 \\
0 & 7 & $\mathrm{Y}$ & 6 & 87.10333 \\
0 & 7 & $\mathrm{Y}$ & 8 & 89.70667 \\
0 & 7 & $\mathrm{Y}$ & 10 & 91.8
\end{tabular}




\begin{tabular}{|c|c|c|c|c|}
\hline 0 & 9 & $\mathrm{~N}$ & 4 & 71.94333 \\
\hline 0 & 9 & $\mathrm{~N}$ & 6 & 74.17333 \\
\hline 0 & 9 & $\mathrm{~N}$ & 8 & 75.61 \\
\hline 0 & 9 & $\mathrm{~N}$ & 10 & 76.82667 \\
\hline 0 & 9 & $\mathrm{Y}$ & 4 & 85.88 \\
\hline 0 & 9 & $\mathrm{Y}$ & 6 & 88.09 \\
\hline 0 & 9 & $\mathrm{Y}$ & 8 & 88.68333 \\
\hline 0 & 9 & $\mathrm{Y}$ & 10 & 92.23333 \\
\hline 0 & 9.5 & $\mathrm{~N}$ & 4 & 68.72667 \\
\hline 0 & 9.5 & $\mathrm{~N}$ & 6 & 70.85 \\
\hline 0 & 9.5 & $\mathrm{~N}$ & 8 & 72.51333 \\
\hline 0 & 9.5 & $\mathrm{~N}$ & 10 & 75.90667 \\
\hline 0 & 9.5 & $\mathrm{Y}$ & 4 & 82.68333 \\
\hline 0 & 9.5 & $\mathrm{Y}$ & 6 & 85.26 \\
\hline 0 & 9.5 & $\mathrm{Y}$ & 8 & 87.70333 \\
\hline 0 & 9.5 & $\mathrm{Y}$ & 10 & 91.50333 \\
\hline 0 & 22 & $\mathrm{~N}$ & 4 & 65.42 \\
\hline 0 & 22 & $\mathrm{~N}$ & 6 & 69.1 \\
\hline 0 & 22 & $\mathrm{~N}$ & 8 & 71.27 \\
\hline 0 & 22 & $\mathrm{~N}$ & 10 & 74.28 \\
\hline 0 & 22 & $\mathrm{Y}$ & 4 & 80.74 \\
\hline 0 & 22 & $\mathrm{Y}$ & 6 & 83.54333 \\
\hline 0 & 22 & $\mathrm{Y}$ & 8 & 85.19333 \\
\hline 0 & 22 & $\mathrm{Y}$ & 10 & 86.29667 \\
\hline 0 & 24 & $\mathrm{~N}$ & 4 & 68.80333 \\
\hline 0 & 24 & $\mathrm{~N}$ & 6 & 69.91667 \\
\hline 0 & 24 & $\mathrm{~N}$ & 8 & 71.23767 \\
\hline 0 & 24 & $\mathrm{~N}$ & 10 & 75.35667 \\
\hline 0 & 24 & $\mathrm{Y}$ & 4 & 82.68 \\
\hline 0 & 24 & $\mathrm{Y}$ & 6 & 85.31667 \\
\hline 0 & 24 & $\mathrm{Y}$ & 8 & 86.58333 \\
\hline 0 & 24 & $\mathrm{Y}$ & 10 & 88.17667 \\
\hline 0 & 25 & $\mathrm{~N}$ & 4 & 68.80333 \\
\hline 0 & 25 & $\mathrm{~N}$ & 6 & 70.74333 \\
\hline 0 & 25 & $\mathrm{~N}$ & 8 & 73.88 \\
\hline 0 & 25 & $\mathrm{~N}$ & 10 & 76.09667 \\
\hline 0 & 25 & $\mathrm{Y}$ & 4 & 83.93 \\
\hline 0 & 25 & $\mathrm{Y}$ & 6 & 84.41333 \\
\hline 0 & 25 & $\mathrm{Y}$ & 8 & 85.63333 \\
\hline 0 & 25 & $\mathrm{Y}$ & 10 & 89.98 \\
\hline 0 & 32 & $\mathrm{~N}$ & 4 & 71.34333 \\
\hline 0 & 32 & $\mathrm{~N}$ & 6 & 73.84 \\
\hline 0 & 32 & $\mathrm{~N}$ & 8 & 73.79 \\
\hline
\end{tabular}




\begin{tabular}{|c|c|c|c|c|}
\hline 0 & 32 & $\mathrm{~N}$ & 10 & 75.51333 \\
\hline 0 & 32 & $\mathrm{Y}$ & 4 & 84.13333 \\
\hline 0 & 32 & $\mathrm{Y}$ & 6 & 87.97 \\
\hline 0 & 32 & $\mathrm{Y}$ & 8 & 89.67333 \\
\hline 0 & 32 & $\mathrm{Y}$ & 10 & 91.56333 \\
\hline 0 & 50 & $\mathrm{~N}$ & 4 & 69.19 \\
\hline 0 & 50 & $\mathrm{~N}$ & 6 & 71.1 \\
\hline 0 & 50 & $\mathrm{~N}$ & 8 & 74.9 \\
\hline 0 & 50 & $\mathrm{~N}$ & 10 & 75.93 \\
\hline 0 & 50 & $\mathrm{Y}$ & 4 & 83.69 \\
\hline 0 & 50 & $\mathrm{Y}$ & 6 & 86.04 \\
\hline 0 & 50 & $\mathrm{Y}$ & 8 & 88.7 \\
\hline 0 & 50 & $\mathrm{Y}$ & 10 & 90.34 \\
\hline 24 & 7 & $\mathrm{~N}$ & 4 & 64.11333 \\
\hline 24 & 7 & $\mathrm{~N}$ & 6 & 66.34667 \\
\hline 24 & 7 & $\mathrm{~N}$ & 8 & 65.73333 \\
\hline 24 & 7 & $\mathrm{~N}$ & 10 & 68.05 \\
\hline 24 & 7 & $\mathrm{Y}$ & 4 & 82.01 \\
\hline 24 & 7 & $\mathrm{Y}$ & 6 & 82.28 \\
\hline 24 & 7 & $\mathrm{Y}$ & 8 & 86.25333 \\
\hline 24 & 7 & $\mathrm{Y}$ & 10 & 86.76667 \\
\hline 24 & 9 & $\mathrm{~N}$ & 4 & 73.02333 \\
\hline 24 & 9 & $\mathrm{~N}$ & 6 & 75.53333 \\
\hline 24 & 9 & $\mathrm{~N}$ & 8 & 77.48333 \\
\hline 24 & 9 & $\mathrm{~N}$ & 10 & 77.50667 \\
\hline 24 & 9 & $\mathrm{Y}$ & 4 & 82.40333 \\
\hline 24 & 9 & $\mathrm{Y}$ & 6 & 82.81333 \\
\hline 24 & 9 & $\mathrm{Y}$ & 8 & 86.27667 \\
\hline 24 & 9 & $\mathrm{Y}$ & 10 & 88.15 \\
\hline 24 & 9.5 & $\mathrm{~N}$ & 4 & 67.56 \\
\hline 24 & 9.5 & $\mathrm{~N}$ & 6 & 69.92667 \\
\hline 24 & 9.5 & $\mathrm{~N}$ & 8 & 72.06667 \\
\hline 24 & 9.5 & $\mathrm{~N}$ & 10 & 83.45333 \\
\hline 24 & 9.5 & $\mathrm{Y}$ & 4 & 83.74333 \\
\hline 24 & 9.5 & $\mathrm{Y}$ & 6 & 85.22 \\
\hline 24 & 9.5 & $\mathrm{Y}$ & 8 & 86.65 \\
\hline 24 & 9.5 & $\mathrm{Y}$ & 10 & 88.53 \\
\hline 24 & 22 & $\mathrm{~N}$ & 4 & 72.73333 \\
\hline 24 & 22 & $\mathrm{~N}$ & 6 & 68.98333 \\
\hline 24 & 22 & $\mathrm{~N}$ & 8 & 71.55333 \\
\hline 24 & 22 & $\mathrm{~N}$ & 10 & 72.70333 \\
\hline 24 & 22 & $\mathrm{Y}$ & 4 & 86.70667 \\
\hline 24 & 22 & $\mathrm{Y}$ & 6 & 87.45333 \\
\hline
\end{tabular}




\begin{tabular}{|c|c|c|c|c|}
\hline 24 & 22 & $\mathrm{Y}$ & 8 & 88.87 \\
\hline 24 & 22 & $\mathrm{Y}$ & 10 & 90.27667 \\
\hline 24 & 24 & $\mathrm{~N}$ & 4 & 71.01 \\
\hline 24 & 24 & $\mathrm{~N}$ & 6 & 76.34667 \\
\hline 24 & 24 & $\mathrm{~N}$ & 8 & 77.4 \\
\hline 24 & 24 & $\mathrm{~N}$ & 10 & 77.72333 \\
\hline 24 & 24 & $\mathrm{Y}$ & 4 & 88.31 \\
\hline 24 & 24 & $\mathrm{Y}$ & 6 & 88.42 \\
\hline 24 & 24 & $\mathrm{Y}$ & 8 & 90.48333 \\
\hline 24 & 24 & $\mathrm{Y}$ & 10 & 90.82 \\
\hline 24 & 25 & $\mathrm{~N}$ & 4 & 67.61667 \\
\hline 24 & 25 & $\mathrm{~N}$ & 6 & 76.34 \\
\hline 24 & 25 & $\mathrm{~N}$ & 8 & 77.33 \\
\hline 24 & 25 & $\mathrm{~N}$ & 10 & 80.83333 \\
\hline 24 & 25 & $\mathrm{Y}$ & 4 & 88.03 \\
\hline 24 & 25 & $\mathrm{Y}$ & 6 & 90.15 \\
\hline 24 & 25 & $\mathrm{Y}$ & 8 & 90.92333 \\
\hline 24 & 25 & $\mathrm{Y}$ & 10 & 91.80333 \\
\hline 24 & 32 & $\mathrm{~N}$ & 4 & 80.90667 \\
\hline 24 & 32 & $\mathrm{~N}$ & 6 & 79.23333 \\
\hline 24 & 32 & $\mathrm{~N}$ & 8 & 81.51 \\
\hline 24 & 32 & $\mathrm{~N}$ & 10 & 82.54333 \\
\hline 24 & 32 & $\mathrm{Y}$ & 4 & 90.47667 \\
\hline 24 & 32 & $\mathrm{Y}$ & 6 & 91.12 \\
\hline 24 & 32 & $\mathrm{Y}$ & 8 & 91.93333 \\
\hline 24 & 32 & $\mathrm{Y}$ & 10 & 93.47667 \\
\hline 24 & 50 & $\mathrm{~N}$ & 4 & 75.77333 \\
\hline 24 & 50 & $\mathrm{~N}$ & 6 & 76.52667 \\
\hline 24 & 50 & $\mathrm{~N}$ & 8 & 76.72667 \\
\hline 24 & 50 & $\mathrm{~N}$ & 10 & 78.59333 \\
\hline 24 & 50 & $\mathrm{Y}$ & 4 & 90.07 \\
\hline 24 & 50 & $\mathrm{Y}$ & 6 & 90.81667 \\
\hline 24 & 50 & $\mathrm{Y}$ & 8 & 92.50333 \\
\hline 24 & 50 & $\mathrm{Y}$ & 10 & 94.91 \\
\hline
\end{tabular}

Table S5. Predictor factor information

\begin{tabular}{llll}
\hline Factor & Type & Levels & Values \\
\hline Grit Size & Fixed & 5 & $240,400,600,800,1200$ \\
Droplet Size $(\mu \mathrm{L})$ & Fixed & 4 & $4,6,8,10$ \\
Si \% & Fixed & 8 & $7.0,9.0,9.5,22.0,24.0,25.0,32.0,50.0$ \\
Time $(\mathrm{hr})$ & Fixed & 2 & 0,24 \\
\hline
\end{tabular}


Table S6. Predictor factor information

\begin{tabular}{|c|c|c|c|}
\hline Factor & Type & Levels I & Values \\
\hline Etching & Fixed & 2 & $\mathrm{~N}, \mathrm{Y}$ \\
\hline Droplet size $(\mu \mathrm{L})$ & Fixed & 4 & $4,6,8,10$ \\
\hline Time (hr) & Fixed & 2 & 0,24 \\
\hline
\end{tabular}

Table S7. Comparing error values for the training and testing set

\begin{tabular}{lll}
\hline Partition & Training & Testing \\
\hline Minimum Error & -9.646 & -5.128 \\
Maximum Error & 6.356 & 10.316 \\
Mean Error & 0.095 & 0.311 \\
Mean Absolute Error & 2.226 & 2.401 \\
Standard Deviation & 2.977 & 3.147 \\
Linear Correlation & 0.933 & 0.901 \\
Occurrences & 96 & 32 \\
\hline
\end{tabular}

